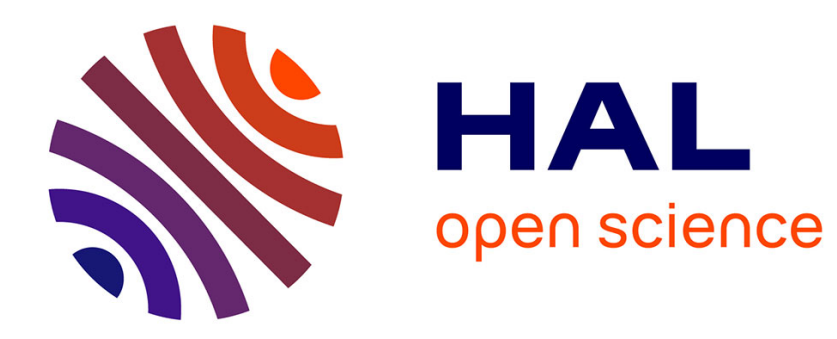

\title{
Origin of shear-induced phase transitions in melts of liquid-crystal polymers \\ Laurence Noirez
}

\section{To cite this version:}

Laurence Noirez. Origin of shear-induced phase transitions in melts of liquid-crystal polymers. Physical Review E , 2004, 10.1103/PhysRevE.72.051701 . hal-01361939

\section{HAL Id: hal-01361939 \\ https://hal.science/hal-01361939}

Submitted on 7 Sep 2016

HAL is a multi-disciplinary open access archive for the deposit and dissemination of scientific research documents, whether they are published or not. The documents may come from teaching and research institutions in France or abroad, or from public or private research centers.
L'archive ouverte pluridisciplinaire $\mathbf{H A L}$, est destinée au dépôt et à la diffusion de documents scientifiques de niveau recherche, publiés ou non, émanant des établissements d'enseignement et de recherche français ou étrangers, des laboratoires publics ou privés. 


\title{
Origin of shear-induced phase transitions in melts of liquid-crystal polymers
}

\author{
Laurence Noirez ${ }^{*}$ \\ Laboratoire Léon Brillouin (CEA-CNRS), Ce-Saclay, 91190 Gif-sur-Yvette Cédex, France
}

(Received 22 December 2004; published 1 November 2005)

\begin{abstract}
Flow induced mechanical properties are often coupled with instabilities, spurt effects, or induced phase transitions. Recent studies have revealed that side-chain liquid crystal polymers exhibit typically shear-induced phases inside the isotropic (nonmesomorphic) liquid state. We present an experimental approach which brings a new understanding for nonlinear flow behaviors. The strategy consists in comparing the critical times issued from the flow behavior of a liquid-crystal polymer to the equilibrium orientational-order relaxation time was characterized. We demonstrate that shear-induced phases do not originate from a flow coupling to conventional orientational order parameter fluctuations. It does not also correspond to a direct coupling with the viscoelastic terminal time, leading to the conclusion that an additional relaxation process takes place with time scales longer than the terminal time. The identification of a low-frequency elastic plateau by viscoelastic measurements corroborates this conclusion.
\end{abstract}

DOI: 10.1103/PhysRevE.72.051701 PACS number(s): 64.70.Md, 47.20.Hw, 82.70.-y, 83.60.Df

\section{INTRODUCTION}

Flow-induced structures are the inevitable product of complex fluid processing and can have dramatic consequences on material properties. These are typically nonlinear effects. The investigation of the molecular processes involved is a key research area and a crucial step for the industrial process optimization.

These phenomena are particularly spectacular when they occur within the isotropic phase. Shear-induced behaviors of this type were essentially observed in giant micellar solutions with the identification of the shear-induced isotropicnematic transition [1,2]. The origin of such phenomena is still a subject of very active debates and developments. Recent studies have revealed that melts of liquid-crystal polymer (LC polymers) exhibit similar shear-induced phase transitions inside the isotropic phase [3,4]. We will use the properties and the knowledge that we have on LC polymers to bring new insights for the understanding of nonlinear phenomena.

The existence of shear-induced phase transitions in LC polymer melts was ascertained by the appearance of a strong birefringence above a critical shear rate; the coexistence of the strong birefringence phase with a weakly birefringent phase; the existence of a stress plateau in the nonlinear flow curve (shear stress versus shear rate); the singularity of the chain conformation [3], which shows the existence of a nonequilibrium conformation different from the conformation at the equilibrium.

How are these different events related? What is the physical meaning of the characteristic times associated to these critical shear values? Is it compatible with predictions? For low molecular weight liquid crystals, predictions say that, starting from a thermodynamically stable isotropic phase, a viscous flow can couple with the orientational-order fluctuations, inducing above a critical shear rate (of about $10^{6} \mathrm{~s}^{-1}$ in

*Email: noirez@1lb.saclay.cea.fr conventional liquid crystals), a nonequilibrium shear-induced phase transitions [1-4].

To answer, at least partly, these questions and predictions, we will focus on a particularly interesting side-chain liquid crystalline polymer of which lifetime $\tau_{\text {fluct }}$ of its orientational-order pretransitional fluctuations was determined by the Kerr effect at equilibrium (without shear) [5].

This is a liquid-crystal polyacrylate of a chemical formula:

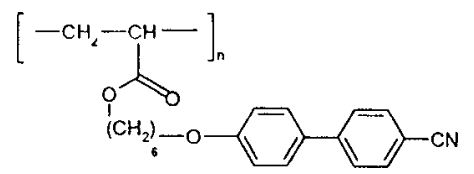

of a molecular weight of 62000 and a polydispersity index of 3.38

It displays the following mesophase sequence: $\mathrm{T}_{\mathrm{g}}$ (glassy state), $\mathrm{N}_{\mathrm{Re}}$ (reentrant nematic), $\mathrm{S}_{\mathrm{Ad}}$ (smectic), $\mathrm{N}$ (nematic), I (isotropic) with an isotropic-nematic transition temperature $\mathrm{T}_{\mathrm{NI}}=131.5^{\circ} \mathrm{C}$ (determined optically by increasing the temperature).

In the equilibrium isotropic phase, previous Kerr measurements [5] carried out on $2240 \mu \mathrm{m}$ thickness samples, have shown that $\tau_{\text {fluct }}$ is about 1000 times larger for the polymer species compared to the low molecular weight homolog. $\tau_{\text {fluct }}$ is diverging in agreement with what was observed for conventional liquid crystals when the temperature approaches $T_{\mathrm{NI}}{ }^{*}$, with, however, an exponent $\beta=1.55$ instead of $1\left[\tau_{\text {fluct }} \alpha\left(T-T_{\mathrm{NI}}^{*}\right)^{-\beta}\right]$. High molecular weight liquid-crystal pretransitional order parameter fluctuations thus disappear more rapidly with temperature.

In the present paper, we will exclusively work with this polymer sample and examine its flow behavior above the isotropic-nematic transition temperature. Using the same polymer, we will confront the equilibrium orientational-order fluctuation time, $\tau_{\text {fluct }}$, from those issued from the nonlinear flow behavior. Three characteristic times thus will be compared: $\tau_{\text {fluct }}, \tau_{\text {term }}$, the viscoelastic characteristic time and $\tau^{*}$ 
$=1 / \gamma^{*}$, the time associated to the critical shear rate of the induced phase. The polymer employed is a polyacrylate chain and the cyano-biphenyl group is a well-known liquid crystal. We trust that this study is representative of the general nonequilibrium behavior of LC polymers. To characterize the flow behavior, rheomicroscopical and viscoelastic measurements have been performed over a large temperature domain above $T_{\mathrm{NI}}$ and using different flow geometries. We will first demonstrate that the characteristic times of the shear-induced phase cannot be coupled to the lifetime $\tau_{\text {fluct }}$ of the order parameter fluctuations measured at the equilibrium. The viscoelastic terminal time $\tau_{\text {term }}$ will be also considered, leading to suppose the extra-long relaxation time scales. The identification of a low frequency elastic plateau will confirm this assumption. We will conclude that, even if the present theoretical models [1-3] (shear banding) well describe the experiments, they are, however, phenomenologically incorrect.

\section{EXPERIMENT}

The rheo-optical observations were performed in transmission mode under crossed-polarized microscopy (Olympus BX60) in the (velocity, neutral axis) plane. The magnification was $100 \mathrm{X}$ and the crossed polarizers were oriented at $45^{\circ}$ with the flow direction. The flow birefringence was ensured using a home-improved CSS450 Linkam shear cell, modified to ensure an optimized thermal environment adapted to study pretransitional phenomena; i.e., a weak temperature gradient $< \pm 0.05^{\circ} \mathrm{C}$. The polymer bulk is placed between the two parallel inner plates of diameter $40 \mathrm{~mm}$. The upper plate is the rotor and the gap is varying from $150 \mu \mathrm{m}$ down to $35 \mu \mathrm{m} \pm 5 \mu \mathrm{m}$. The monochromatic light was produced using a photoluminescent diode of wavelength $\lambda=4700 \AA \pm 150 \AA$. The birefringence was determined using the relationship: $I / I_{0}=\sin ^{2}(\Delta n e \pi / \lambda)$, where $e$ is the plateplate gap and $I$ is the transmitted intensity and $I_{0}$ the uncrossed polarizer intensity.

Viscoelastic measurements were performed with a rheometer (ARES) equipped with an air-pulsed oven. This thermal environment ensures a temperature control within a $0.1{ }^{\circ} \mathrm{C}$ interval. The shear geometry was an aluminum cone and plate cell (diameter: $12 \mathrm{~mm}$, angle: $2.25^{\circ}$ ) in dynamic frequency sweep mode.

\section{RESULTS AND DISCUSSION}

The evolution of the flow birefringence has been studied as a function of the temperature and of the shearing thickness. As already observed in other LC polymers $[3,4]$, this polymer presents a strong nonlinear behavior with, above a critical shear rate, an abrupt birefringence increase. Figure 1 illustrates the nonlinear evolution of the birefringence from $\Delta T=+0.2{ }^{\circ} \mathrm{C}$ to $\Delta \mathrm{T}=+8{ }^{\circ} \mathrm{C}$ above $T_{N I}$ for a gap thickness of $100 \mu \mathrm{m}$. Two regimes can be distinguished; a low shear rate regime and a strongly shear-rate-dependent regime (it seems also that at a very high shear rate and close to the transition, $\Delta n$ diplays a plateaulike onset). The low shear rate regime depends on the temperature but weakly on the shear

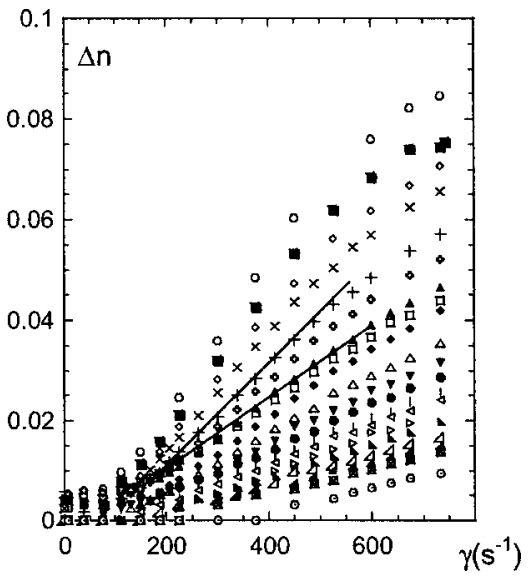

FIG. 1. Evolution of the birefringence $\Delta n$ as a function of the shear rate displayed by the LC polymer at various temperatures above the equilibrium isotropic-nematic temperature. The gap thickness is $100 \mu \mathrm{m}$ (plate-plate shear geometry). The observation is carried out in the (velocity, neutral axis) plane between crossed polarizers oriented at $45^{\circ}$ from the velocity axis $\left(T_{N I}=131.5^{\circ} \mathrm{C}\right)$. $\bigcirc, 132{ }^{\circ} \mathrm{C} ;+, 133{ }^{\circ} \mathrm{C} ; \quad, 134^{\circ} \mathrm{C} ; \mid, 135.6^{\circ} \mathrm{C} ; \Delta, 137.5^{\circ} \mathrm{C} ; \mathbf{\square}$, $132.2^{\circ} \mathrm{C} ; \boldsymbol{\Delta}, 133.6^{\circ} \mathrm{C} ; \triangle, 134.4^{\circ} \mathrm{C} ; \triangleleft, 136^{\circ} \mathrm{C} ; \boldsymbol{\Lambda}, 138^{\circ} \mathrm{C} ; \vartheta$, $132.4{ }^{\circ} \mathrm{C} ; \mathbf{\square}, 133.2^{\circ} \mathrm{C} ; \boldsymbol{\nabla}, 134.8^{\circ} \mathrm{C} ; \triangleright, 136.5^{\circ} \mathrm{C} ; \otimes, 138.5^{\circ} \mathrm{C} ; \times$, $132.6^{\circ} \mathrm{C} ; \square, 133.8^{\circ} \mathrm{C} ; \boldsymbol{O}, 135.2^{\circ} \mathrm{C} ; \boldsymbol{\nabla}, 1370^{\circ} \mathrm{C} ; \mathbf{\square}, 140^{\circ} \mathrm{C}$.

rate, defining a shear rate interval from $0.1 \mathrm{~s}^{-1}$ up to about $150 \mathrm{~s}^{-1}$ corresponding to a birefringence plateau with respect to the second regime. This first regime can be assimilated to the conventional flow effect on the pretransitional fluctuations [10]. It corresponds to the paranematic phase [6-8]. In this regime, $\Delta n$ is a linear function of the shear rate. According to the low-shear-rate model [10], the flow-induced birefringence is suppose to increase slightly the more the temperature is close to $\mathrm{T}_{\mathrm{NI}}$. This description is consistent with our observation of the first regime and in agreement with the observations carried on small liquid-crystal molecule [9]. One can also notice that the value at zero shear is not zero. This can be explained by the contribution of the pretransitional fluctuations giving rise to a non-negligible scattered intensity. The second regime is defined by an abrupt departure of the birefringence above a critical shear rate as predicted by the different theories [1-4]. This regime cannot be explained on the simple basis of the usual linear approximation. It corresponds to a strong discontinuity in the rheooptical behavior and reveals the appearance of a nonequilibrium first order phase transition. As already observed in other LC polymers, this nonlinear behavior is characterized by a alignment of the director along the velocity axis and a texture free from defects. It indicates a nonequilibrium nematic phase. In a first approximation, this regime could be modeled by this relationship: $\Delta n=K(T)\left[\gamma-\gamma^{*}(T)\right]$ with $\gamma^{*}(T)$ the critical shear rate and $K(T)$ a temperature-dependent constant which measures the elastic coupling to the shear stress. Figure 2 displays the evolution of $\gamma^{*}(T)$ versus temperature. It shows that the critical shear rate increases notably with increasing temperature following an approximate linear dependence from which a pseudo-second-order nonequilibrium critical temperature $T_{N I}^{*}$ can be extracted: $\gamma^{*}(T) \alpha\left(T-T_{N I}^{*}\right)$. The extrapolation gives $T_{N I}^{*}=130 \pm 1{ }^{\circ} \mathrm{C}$ (against $T_{\mathrm{NI}}$ 


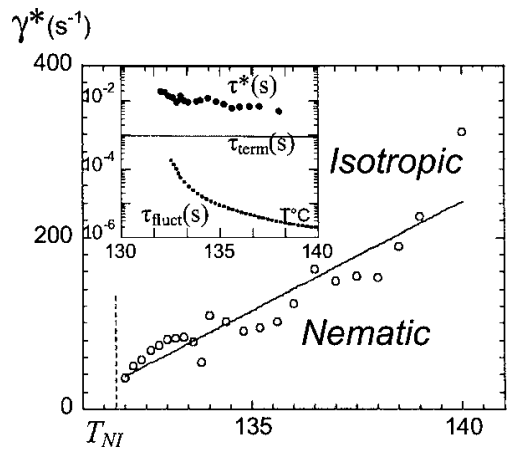

FIG. 2. Plot of the critical shear rate $\gamma^{*}$ as a function of the temperature. In the inset are reported versus temperature (logarithmic scale): the critical shear rate inverse $\tau^{*}(\mathbf{O})=1 / \gamma^{*}$; the equilibrium orientational-order lifetime $\tau_{\text {fluct }}($ ) (from Ref. [5]); the viscoelastic relaxation $\tau_{\text {term }}(-)$.

$=131.5 \pm 0.1^{\circ} \mathrm{C}$ for the equilibrium transition temperature). This is the behavior expected for a nonequilibrium first order transition, which assumes a direct flow coupling with orientational-order fluctuations. However, these shear rates do not fit at all with the lifetime $\tau_{\text {fluct }}$ of the static orientational-order fluctuations, $\tau_{\text {fluct }}$ is indeed about $10^{3}$ times shorter, at $1{ }^{\circ} \mathrm{C}$ above $T_{N I}$, than the time deduced from the critical shear rate $\tau^{*}=1 / \gamma^{*}$ (inset of Fig. 2). Of course, this difference becomes even larger at higher temperatures above $T_{\mathrm{NI}}$.

The discrepancy between the critical shear rate corresponding to the appearance of the shear-induced phase and the lifetime of the orientational-order fluctuations is significant. Could it be an artefact result due to a slip regime? Wall slip is expected when the sheared melt displays no more viscous properties. An easy method of identifying slip transition is to vary the shear gap. Figures 3 display the evolution of the birefringence as a function of the shear rate from $35 \mu \mathrm{m}$ up to $150 \mu \mathrm{m}$ gap thickness, at $\Delta T=+1{ }^{\circ} \mathrm{C}$ above the isotropic-nematic transition temperature. The two series of measurements corresponding to Figs. 3(a) and 3(b), respectively, have been carried out independently. Since the gap values of the two series overlap, the whole measurements cover gap thicknesses from $35 \mu \mathrm{m}$ up to $150 \mu \mathrm{m}$. The superposition of the birefringence values (except partly at $35 \mu \mathrm{m}$ - the error bars being higher because of the few polymer amount) demonstrates that the shear-induced birefrin-

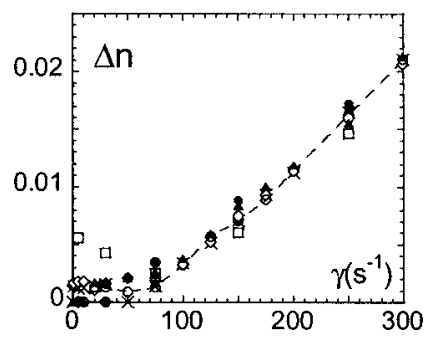

FIG. 3. Influence of the gap thickness on the shear-induced birefringence measured in the (velocity, neutral axis) plane, at $\Delta \mathrm{T}=$ $+1{ }^{\circ} \mathrm{C}$ above the isotropic-nematic transition temperature: $150 \mu \mathrm{m}$ $(\times), 125 \mu \mathrm{m}(\diamond), 100 \mu \mathrm{m}(\bigcirc), 50 \mu \mathrm{m}(\bullet), 75 \mu \mathrm{m}(\boldsymbol{\Delta})$, and $35 \mu \mathrm{m}(\square)$.

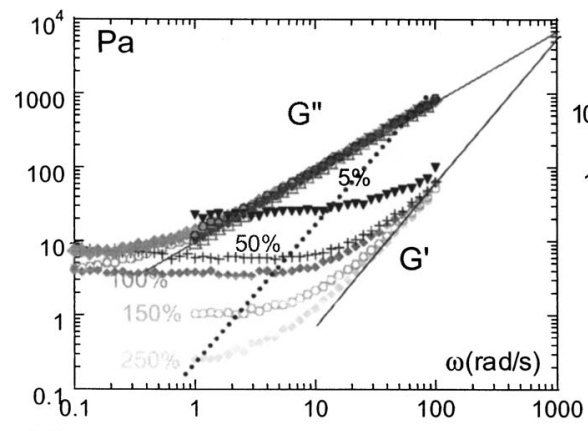

(a)

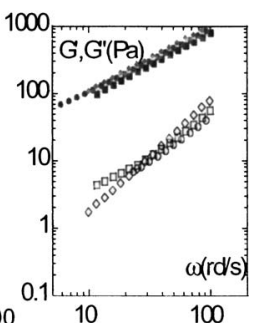

(b)
FIG. 4. (Color online) (a) Frequency dependence of the viscoelastic moduli $\left[\mathrm{G}^{\prime}(\omega)\right.$ and $\left.\mathrm{G}^{\prime \prime}(\omega)\right]$ of the LC polymer measured at $\Delta \mathrm{T}=+5^{\circ} \mathrm{C}$ above the isotropic-nematic transition temperature, with a cone-plate geometry (diameter: $12 \mathrm{~mm}$, angle: $2.25^{\circ}$ ) at different strain amplitudes [5\%: $\mathrm{G}^{\prime}(\boldsymbol{\nabla}), \mathrm{G}^{\prime \prime}(\bigcirc) ; 50 \%: \mathrm{G}^{\prime}(+), \mathrm{G}^{\prime \prime}$ $(\diamond) ; 100 \%: G^{\prime}(), G^{\prime \prime}\left(\right.$ ); $150 \%: G^{\prime}(), G^{\prime \prime}(\nabla) ; 250 \%: G^{\prime}(>), G^{\prime \prime}$ (ए)]. The solid lines correspond to the $\omega$ and $\omega^{2}$ curves. The dotted $\omega^{2}$ line points out the flow behavior coinciding with the time scale of the phase transitions induced from above $\mathrm{T}_{\mathrm{NI}}$ up to $135^{\circ} \mathrm{C}$ [fitting with $\gamma^{*}<100 \mathrm{~s}^{-1}$ (Fig. 2)]. 4(b) Temperature dependence of the viscoelastic moduli; overlaying of the dynamic spectra determined in a strain regime independent of its amplitude at: $130{ }^{\circ} \mathrm{C}$ [ $\left.\mathrm{G}^{\prime}(\diamond), \mathrm{G}^{\prime \prime}(\diamond)\right], 135^{\circ} \mathrm{C}\left[\mathrm{G}^{\prime}(\square), \mathrm{G}^{\prime \prime}(\boldsymbol{\square})\right]$, and $147^{\circ} \mathrm{C}\left[\mathrm{G}^{\prime}(\bigcirc)\right.$, $\left.\mathrm{G}^{\prime \prime}(\mathbf{O})\right]$.

gence is a bulk property, independent of the sheared thickness. This study as a function of the thickness illustrates also very well the abrupt character of the isotropic-nematic transition which occurs clearly above a critical value [Figs. 3(a) and 3(b)]. It should be noted that if slip transition would have taken place, the discrepancy between the critical shear rate and the orientational-order fluctuation lifetime would have been even higher. The lifetime of the orientationalorder fluctuations is not reached whereas the phase is already shear induced. The nonequilibrium phase is definitively not due to the static orientational pretransitional fluctuations. It is consequently necessary to compare with other times in the system. Let us determine the terminal relaxation time of the melt, $\tau_{\text {term }}$.

We report here viscoelastic studies carried out on the same polymer. We will show that the viscoelastic results corroborate the birefringence conclusions. The present results have been measured using the cone-plate geometry. Similar data have been obtained at a $0.8 \mathrm{~mm}$ gap with a plate-plate geometry. The viscoelastic measurements have been performed at different temperatures above $T_{N I}$. Figure 4 displays the frequency dependence of the storage $\mathrm{G}^{\prime}(\omega)$ and the loss $\mathrm{G}^{\prime \prime}(\omega)$ moduli at $\Delta T=+5{ }^{\circ} \mathrm{C}$ at different strain amplitudes. The evolution of the viscoelastic moduli in Fig. 4 indicates a nontrivial behavior characterized by the existence of two different frequency regimes. At low frequency, the invariance of $\mathrm{G}^{\prime}$ defines an elastic plateau. Its evolution with the strain amplitude indicates that this low-frequency domain corresponds to a nonlinear response. The large strain rates produce a lowering of the elastic plateau making a classical polymer melt behavior at high frequency emerged. At large strain amplitudes and high frequency, $\mathrm{G}^{\prime \prime}(\omega)$ and $\mathrm{G}^{\prime}(\omega)$ val- 
ues are independent of the strain, defining a regime of linear deformation. The terminal time $\tau_{\text {term }}$ is determined in the thermal conditions of the shear-induced phase exploring the frequency interval $(50 \leqslant \omega \leqslant 100 \mathrm{rad} / \mathrm{s})$, which presents a linear response at high strain. This frequency interval is characterized by $\mathrm{G}^{\prime}(\omega)$ approaching $\omega^{2}$ scaling (yielding the zero-shear viscosity) and $\mathrm{G}^{\prime \prime}(\omega)$ fitting with $\omega$ scaling. At this temperature and within the observation frequency window, the LC polymer behaves as an ordinary flexible polymer bulk far above the glassy temperature Tg. The extrapolated terminal time is given by the intersection of the $\omega$ and $\omega^{2}$ scaling lines $\tau_{\text {term }} \cong 10^{-3} \mathrm{~s}$. This value is consistent with other terminal times $\left[\tau_{\text {term }}(\mathrm{PMA}) \approx 10^{-3} \mathrm{~s}\right]$ determined in the isotropic phase at $75^{\circ} \mathrm{C}$ above the glassy temperature, on LC polymethacrylates of equivalent weight average polymerization degrees $[11,12]$. To compare the terminal time to the time scale of the shear-induced phase, we plot in Fig. 4(a) (dotted line), the $\omega^{2}$ scaling that would coincide to the time scale of shear-induced phase transitions within $\mathrm{T}_{\mathrm{NI}}<\mathrm{T}<135^{\circ} \mathrm{C}$ [i.e., fitting with $\gamma^{*}<100 \mathrm{~s}^{-1}$ (Fig. 2)]. The gap between the two $\omega^{2}$ scaling estimates the difference between the time scales. The terminal time $\tau_{\text {term }}$ is shorter. The shear-induced phase does not originate from a flow coupling with the viscoelastic properties of the melt since the phase is induced before reaching the terminal time. Moreover, $\gamma^{*}$ displays a critical behavior approaching asymptotically the isotropicnematic transition temperature (inset of Fig. 2) which is not consistent with the classical temperature dependence of a terminal time. Indeed, for a nonentangled polymer, the characteristic time obeys the Rouse dynamics and varies as a function of the temperature as the inverse: $\tau_{\text {Polymer }}$ $=\tau_{\text {Rouse }} \alpha \eta M / R T$. Experimentally, the viscoelastic moduli are almost superimposed [Fig. 4(b)] in a range of $15^{\circ} \mathrm{C}$ over the isotropic-nematic phase transition, demonstrating that $\tau_{\text {term }}$ and $\tau^{*}$ cannot intercept.

Finally, the very unsettled elastic plateau $\mathrm{G}^{\prime}(\omega)$ found at low frequency suggests that the material contains cooperative rearrangements as in rubbered polymers. The nonlinear character of the plateau behavior indicates a dissipative response of this "solidlike" response with increasing strain. The invariance of $\mathrm{G}^{\prime \prime}(\omega)$ fitting with $\omega$ scaling down to $1 \mathrm{rad} / \mathrm{s}$ indicates that the material contains also a viscous component. A series of experiments is on the way to extend the study and the analysis to different viscoelastic materials [13]. The nonlinear behavior of the birefringence should thus be related to the rearrangement of these macroscopic elastic forces, which include orientational-order fluctuations of whose time scales can be dramatically slowed down (and thus become undetectable via conventional Kerr measurements).

\section{CONCLUSIONS}

These experimental observations drive to the conclusion that a classical approach (coupling with the conventional orientational-order fluctuation life time or the viscoelastic terminal time) is not suitable to elucidate the origin of shearinduced phase transitions. It appears now clearly that the flow is coupled with an additional longer relaxation time scale more compatible with the existence of a threedimensional structuration of the material as indicated by the macroscopic dynamic elastic plateau response. This conclusion is also coherent considering other recent results mainly reported on LC polymers. Stretching experiments were carried out on on nonentangled side-chain LC polymers in the nematic phase [14]. The possibility of stretching is phenomenologically very intriguing. Clearly, a connectivity between chains in the nematic phase provides the necessarily cohesion to allow the stretching. On the other hand, anterior viscoelastic measurements carried out on thin films with a piezorheometer have revealed the existence of elastic heterogeneities within the isotropic melt [15]. These measurements show that the viscoelastic behavior passes continuously from a classical viscoelastic liquidlike to a solidlike behavior by decreasing the thickness from $120 \mu \mathrm{m}$ down to $\sim 10 \mu \mathrm{m}$. Further studies on nonentangled Polystyrene chains have revealed similar features [16]. These heterogeneities should not be related to a mesomorphic property. The authors conclude to the existence of ten micrometer sized density fluctuations (clusters), which are frozen at the time scales of common experiments and which should be associated to the glassy transition.

Our results actually indicate that the frozen state is extended to the sample size and not restricted to density fluctuations. The shear-induced phase can be understood as resulting from frozen pretransitional fluctuations, which are not measurable by Kerr effects. When the shear flow interplays with these fluctuations embedded in the dynamic clusters, the entrance in the nonlinear regime occurs much earlier compared to a coupling with the life time of the orientational-order fluctuations determined by Kerr effects or with the viscoelastic terminal time. This is what is observed.

In this view, because of the established elastic character of LC polymers, it seems that models developed within the frame of nematic elastomers [17-19] could be helpful in describing the rheological behavior of liquid crystalline melts even if conventional entanglements are absent.

\section{ACKNOWLEDGMENTS}

We are very grateful to P. Martinoty, who provided us with the sample used to carry out the Kerr effect measurements. 
[1] V. Schmitt, F. Lequeux, A. Pousse, and D. Roux, Langmuir 10, 955 (1994).

[2] J. F. Berret, D. C. Roux, and G. Porte, P. Linder, Europhys. Lett. 25, 521 (1994).

[3] C. Pujolle-Robic and L. Noirez, Nature (London) 409, 167 (2001).

[4] C. Pujolle-Robic, P. D. Olmsted, and L. Noirez, Europhys. Lett. 59, 364 (2002).

[5] V. Reys, Y. Dormoy, J. L. Gollani, P. Martinoty, P. LeBainy, and J. Dubois, Phys. Rev. Lett. 61, 2340 (1988).

[6] S. Hess, Z. Naturforsch. 31a, 1507 (1976); P. Igl and S. Hess, Rheol. Acta 44, 465 (2005).

[7] A. Onuki and K. Kawasaki, Ann. Phys., 121, 456 (1979).

[8] P. D. Olmsted and P. Goldbart, Phys. Rev. A 41, R4578 (1990).

[9] H. Pleiner, M. Liu, and H. R. Brand, Rheol. Acta 41, 375 (2002).

[10] P. G. de Gennes and J. Prost, The Physics of Liquid Crystals
(Oxford Science Publications, Oxford, 1993).

[11] R. H. Colby et al., Liq. Cryst. 13, 233 (1993).

[12] S. F. Rubin, R. M. Kannan, J. A. Kornfield, and C Boeffel, Macromolecules 28, 3521 (1995).

[13] H. Mendil, P. Baroni, and L. Noirez, Europhys, Lett. (to be published).

[14] V. Fourmeaux-Demange et al., Eur. Biophys. J. E1, 301 (2000).

[15] J. L. Gallani, L. Hilliou, P. Martinoty, and P. Keller, Phys. Rev. Lett. 72, 2109 (1994); P. Martinoty, L Hilliou, M. Mauzac, L Benguigui, and D. Collin, Macromolecules 32, 1746 (1999).

[16] D. Collin and P. Martinoty, Physica A 320, 235 (2002).

[17] M. Warner, K. P. Gelling, and T. A. Vilgis, J. Chem. Phys. 88, 4008 (1988).

[18] A. Halperin, J. Chem. Phys. 85, 1081 (1988).

[19] R. Sigel, W. Stille, G. Strobl, and R. Lehnert, Macromolecules 26, 4226 (1993) 